\title{
Zoonosemonitoring bei Schlachtschweinen mittels analytischer Mikroarrays
}

\author{
Verena K. Meyer ${ }^{1}$, Klaus Wutz ${ }^{1}$, Piotr Krol' ${ }^{2}$ Silke Wacheck ${ }^{2}$, Manfred Gareis ${ }^{2}$, Christina Nölting ${ }^{3}$, \\ Friedhelm Struck ${ }^{3}$, Erwin Soutschek ${ }^{3}$, Oliver Böcher ${ }^{3}$, Reinhard Niessner ${ }^{1}$, Michael Seidel ${ }^{1}$ \\ ${ }^{1}$ Institut für Wasserchemie und Chemische Balneologie, Lehrstuhl für Analytische Chemie, \\ Technische Universität München, Marchioninistr. 17, 81377 München, Deutschland \\ verena.meyer@tum.de \\ ${ }^{2}$ Lehrstuhl für Lebensmittelsicherheit, Tierärztliche Fakultät, Ludwig-Maximilians-Universität München, \\ Schönleutnerstr. 8, 85764 Oberschleißheim, Deutschland \\ ${ }^{3}$ Mikrogen GmbH, Floriansbogen 2-4, 82061 Neuried, Deutschland
}

\begin{abstract}
:
Zoonosen sind Infektionskrankheiten, die von Tieren auf den Menschen übertragen werden können. Bei Mastschweinen und Schweinefleischprodukten stellen die Bakterien Campylobacter spp., Salmonella spp. und enteropathogene Yersinia spp., das Hepatitis-E-Virus und die Parasiten Trichinella spp. und Toxoplasma spp. relevante Erreger dar.

Zur Warenkontrolle und um den Hygienestatus eines Betriebes zu erfassen wird ein Verfahren benötigt, mit dessen Hilfe eine Probe schnell, kostengünstig und gleichzeitig auf verschiedene Erreger hin untersucht werden kann.

Mittels des hier vorgestellten Antigen-Mikroarray-Immunoassays ist der parallele Nachweis Erregerspezifischer Antikörper ( $\mathrm{lgG}$ ) in Serum- oder Fleischtropfsaftproben möglich.

Am Beispiel des Hepatitis-E-Virus und Yersinia spp. wurde der Mikroarray-Immunochip mithilfe eines Seren-Panels bezüglich analytischer Parameter charakterisiert und mit den Alternativmethoden recomLine HEV (Human-Test, adaptiert für Schweineseren, Mikrogen, Neuried) beziehungsweise pigtype Yersinia Ab (Qiagen Leipzig, Leipzig) und einem herkömmlichen Mikrotiterplatten-ELISA verglichen. Der Immunochip erreichte in Verdünnungsexperimenten jeweils die höchste Sensitivität und Nachweisstärke. 76\% der Seren wurden mit dem Line-Immunoassay positiv auf HEV-Antikörper getestet. Mit dem ELISA und dem Immunochip hingegen wurden anti-HEV-IgG in 93\% und 90\% der Proben nachgewiesen. Das Screening für anti-Yersinia-lgG führte mit dem kommerziell erhältlichen ELISA-Kit zu 45\% positiven Ergebnissen, während 59\% der Serumproben mit dem selbst entwickelten ELISA und $86 \%$ mit dem Immunochip als positiv befunden wurden.
\end{abstract}

Mithilfe des Mikroarrays konnten Antikörper gegen HEV und Yersinia spp. zuverlässig unterschieden und parallel nebeneinander nachgewiesen werden.

Key words: Zoonosen, Antigen-Immunoassay, Chemilumineszenz-Detektion, MCR 3

\section{Einleitung}

Bei der Nahrungsmittelherstellung werden insbesondere an Produkte tierischer Herkunft hohe Maßstäbe bezüglich der Produktsicherheit und der Prozesshygiene angelegt. Durch ein effizientes Monitoring relevanter ZoonoseErreger soll deren Eintrag in die Lebensmittelkette signifikant verringert werden, um lebensmittelhygienisch bedingten Erkrankungen vorzubeugen und so dem Verbraucherschutz gerecht zu werden. Bisher routinemäßig angewandte Analyseverfahren beruhen auf dem vergleichsweise zeit- und kostenintensiven Nachweis einzelner
Zielpathogene. Mithilfe hochparalleler Verfahren, wie z. B. ChemilumineszenzMikroarray-Immunoassays (CL-MIA), können mehrere Pathogene gleichzeitig untersucht und Analysezeiten sowie Kosten deutlich reduziert werden. Wird dabei ein Antigen-Mikroarray zum Nachweis Erreger-spezifischer Antikörper eingesetzt, so werden auch zurückliegende Infektionen mit erfasst und die Seroprävalenz von Tierbeständen kann bestimmt werden.

Mithilfe des hier entwickelten AntigenMikroarray-Immunoassays können Serum- und Fleischtropfsaftproben von geschlachteten Schweinen auf Antikörper gegen relevante 
Zoonose-Erreger untersucht werden. Dazu werden auf einer Polyethylenglycolmodifizierten Glasoberfläche [1] Erregerspezifische rekombinante oder extrahierte Antigene immobilisiert. Die aus der verdünnten Serumprobe daran gebundenen ZoonoseAntikörper werden mithilfe von Enzymmarkierten Detektionsantikörpern nachgewiesen, die eine ChemilumineszenzReaktion katalysieren. Die Auslesung der generierten Chemilumineszenz-Signale erfolgt mittels einer CCD-Kamera. Das Assay-Prinzip ist in Abbildung 1 schematisch dargestellt.

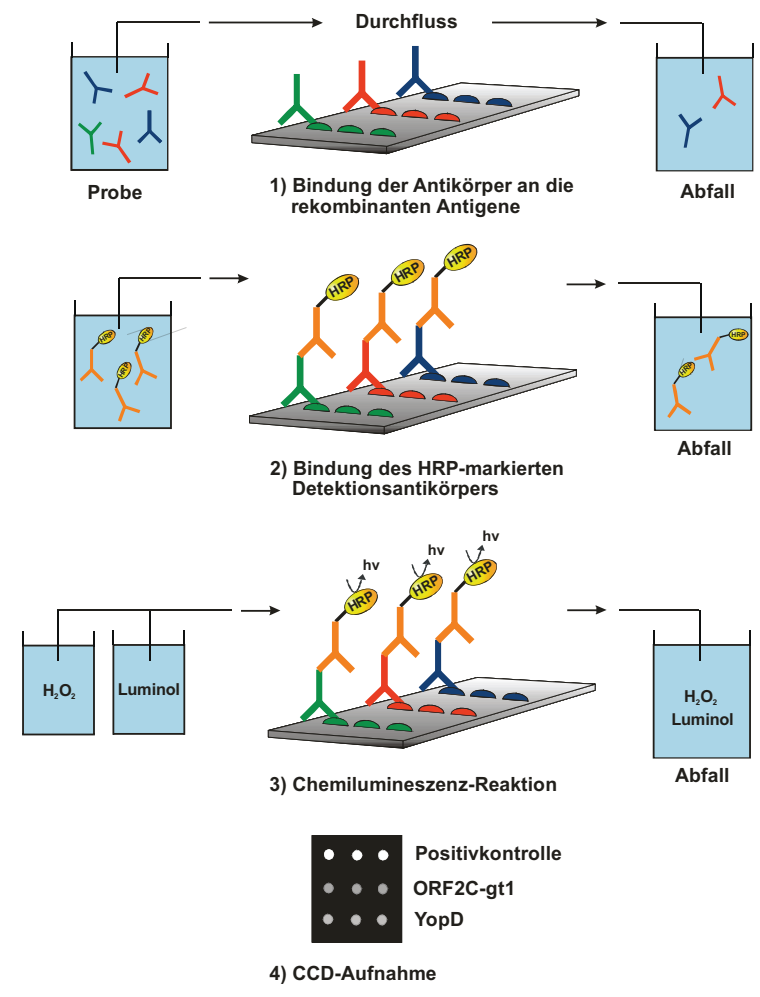

Abbildung 1. Schematische Darstellung des CL-MIAPrinzips mit rekombinanten Antigenen.

Das gesamte Messverfahren wird auf der am Lehrstuhl für Analytische Chemie entwickelten vollautomatischen Analyse-Plattform MCR 3 (siehe Abbildung 2) durchgeführt.

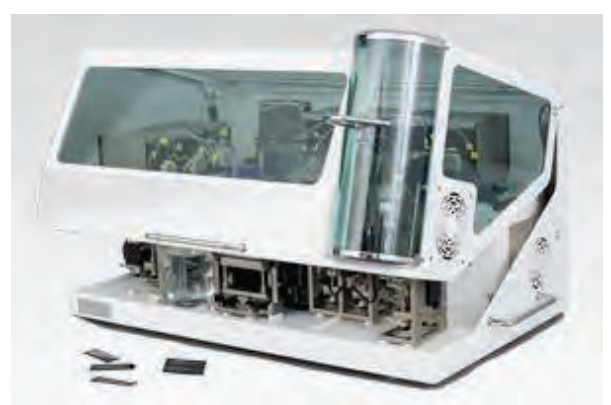

Abbildung 2. Mikroarray-Analyse-Plattform MCR 3.
Diese stellt ein fluidisches System dar und ermöglicht damit im Vergleich zu stationären Immunoassays deutlich kürzere Messzeiten [2].

Durch den Einsatz dieser innovativen Methode können in einer Probe innerhalb weniger Minuten relevante Zoonose-Antikörper parallel und kostengünstig nachgewiesen werden [3].

\section{Materialien und Methoden}

Die verwendeten Glasobjektträger wurden silanisiert sowie mit DAPEG und Diepoxy-PEG beschichtet, um die rekombinanten Antigene kovalent zu binden. Die verschiedenen Antigene wurden mithilfe des Mikrokontaktdruckers BioOdyssey Calligrapher MiniArrayer (Bio-Rad Laboratories, München) in Reihen nebeneinander aufgebracht. Um die freien Bindungsstellen auf der Oberfläche zu inaktivieren, wurden die belegten Glaschips mit TRIS-HCl-Puffer ( $\mathrm{pH} 7,8)$ und mit 1\%-iger BSALösung behandelt. Die Glaschips wurden über eine doppelseitige Klebefolie an einen Kunststoffträger gekoppelt, der zur Anbindung an die Fluidik der vollautomatischen MikroarrayAnalyseplattform MCR 3 (Munich Chip Reader, gwk Präzisionstechnik, München) diente.

Für die Assay-Entwicklung wurden von der Fa. Mikrogen (Neuried) die rekombinanten Antigene ORF2C-gt1 und ORF2C-gt3 des Hepatitis-E-Virus sowie YopD zum Nachweis von Antikörpern gegen Yersinia spp. zur Verfügung gestellt. Die untersuchten Schweineserumproben stammten von bayerischen Schlachthöfen und wurden vor der Messung 1:100 mit PBST (10 mM KH $\mathrm{KO}_{4}$, $70 \mathrm{mM} \mathrm{K} \mathrm{K}_{2} \mathrm{HPO}_{4}, 145 \mathrm{mM} \mathrm{NaCl}, 0,05 \%$ (v/v) Tween ${ }^{\circledR}-20$ ) verdünnt. Das pro Messung benötigte Volumen der verdünnten Probe betrug $1 \mathrm{~mL}$. Als Laufpuffer wurde PBST verwendet. Die Detektion beruhte auf einer HRP-katalysierten Chemilumineszenz-Reaktion der Substrate Luminol und Wasserstoffperoxid (WESTAR SUPERNOVA ELISA, Cyanagen, Bologna, Italien), deren Intensität mittels einer CCD-Kamera aufgezeichnet wurde.

\section{Ergebnisse und Diskussion}

Zunächst wurde die Reproduzierbarkeit des Messsignals eines deutlich seropositiven Serums auf mehreren Chips und an drei verschiedenen Messtagen untersucht. Für ORF2C-gt1 ist mit einem Variationskoeffizient von $6,6 \%$ bei 33 Messungen an drei Tagen eine hohe Reproduzierbarkeit gegeben. Für YopD betrug der Variationskoeffizient bei 16 Messungen an drei Tagen 11,8\%. 
Anhand der entsprechenden Mehrfachmessung eines seronegativen Serums wurde jeweils ein Entscheidungswert (Cut-off) ermittelt, ab dem eine Probe als seropositiv einzustufen ist.

Ausgehend von einer 1:100-Verdünnung (relative Serumkonzentration 100\%) wurden jeweils mit einem stark positiven Serum Verdünnungsreihen angesetzt und mithilfe des entwickelten CL-MIA, einem herkömmlichen ELISA sowie einem kommerziell erhältlichen Testkit analysiert.

Hinsichtlich der Sensitivität ist der CL-MIA für ORF2C-gt1 dem Mikrotiterplatten-ELISA und besonders dem für Schweineseren adaptierten recomLine-Test zur Humandiagnostik deutlich überlegen. So erstreckt sich der lineare Bereich (relative Serumkonzentration $100 \%$ bis $25 \%$ ) für den CL-MIA etwa vom 17-fachen bis zum vierfachen Cut-off-Wert, für den ELISA nur vom siebenfachen bis zum dreifachen und für den recomLine-Test gar nur vom vierfachen bis zum zweifachen Cut-off-Wert. Darüber hinaus ist auch das relative Nachweisvermögen der selbst entwickelten Methoden höher. Während für den adaptierten recomLine-Test bereits bei einer Verdünnung von 1:40 der Cut-off-Wert unterschritten wird, erfolgt dies für den CL-MIA und den Mikrotiterplatten-ELISA erst bei einer Verdünnung von 1:100.

Für YopD weist der CL-MIA eine signifikant höhere Sensitivität im Vergleich zu den beiden ELISA-Methoden auf. Während sich der lineare Bereich (relative Serumkonzentration $100 \%$ bis $25 \%$ ) für den CL-MIA etwa vom 43-fachen bis zum 14-fachen Cut-off-Wert erstreckt, liegt er für den selbst entwickelten ELISA und den pigtype Yersinia $\mathrm{Ab}$ lediglich zwischen dem drei- beziehungsweise vierfachen und dem zweifachen Cut-off-Wert. Messtechnisch zu einem seronegativen Befund gelangt man mit dem CL-MIA ab einer Verdünnung von 1:400, mit den anderen beiden Methoden hingegen schon ab einer Verdünnung von 1:40. Somit ist der CL-MIA das nachweisstärkste Verfahren.

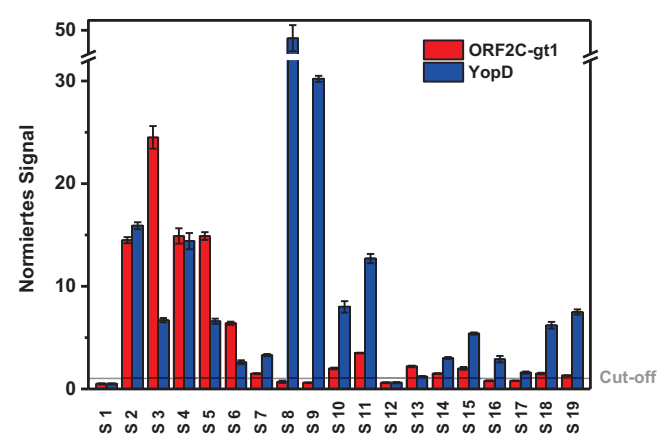

Abbildung 3. Parallele Detektion von HEV- und Yersinia-Antikörpern mittels ORF2C-gt1 und YopD.
Um die Multianalyt-Fähigkeit des Mikroarrays darzulegen, wurden einige Seren gleichzeitig auf Antikörper gegen HEV und Yersinia spp. hin untersucht (siehe Abbildung 3). Die normierten Signalintensitäten wie auch die seropositiven und seronegativen Befunde treten für die beiden rekombinanten Antigene unabhängig voneinander auf (siehe auch Abbildung 4). So finden sich Seren, die mit beiden Antigenen nicht reagieren (z. B. S 1) oder deutlich mit beiden Antigenen reagieren (z. B. S 2), wie auch solche Seren, die nur gegen einen der beiden Erreger Antikörper enthalten (z. B. S 9 und $S$ 13).

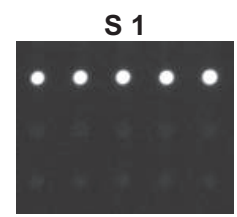

S 9

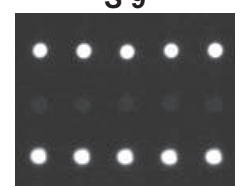

S 2

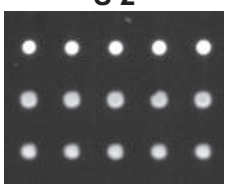

Positivkontrolle

ORF2C-gt1

YopD

S 13

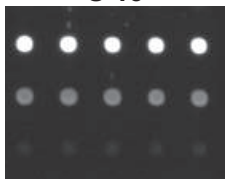

Positivkontrolle ORF2C-gt1

YopD
Abbildung 4. CCD-Aufnahmen der am MCR 3 gemessenen Seren S 1, S 2, S 9 und S 13.

Anhand des Antigens ORF2C-gt1 wurde die Lagerfähigkeit des fertigen Chips über zwei Wochen beziehungsweise zwei Monate bei verschiedenen Lagertemperaturen $\left(-20^{\circ} \mathrm{C}, 4^{\circ} \mathrm{C}\right.$ und Raumtemperatur) untersucht (siehe Abbildung 5).

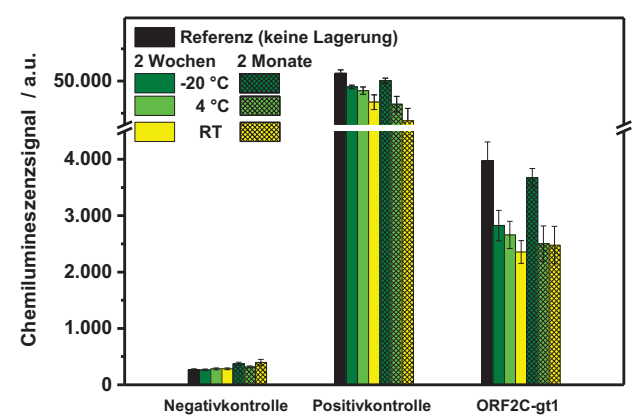

Abbildung 5. Untersuchung zur Lagerstabilität.

Dabei lieferte eine Lagerung bei $-20^{\circ} \mathrm{C}$ die höchsten Chemilumineszenzsignale. Zusätzlich verschlechterte sich die Spotqualität bei höheren Lagertemperaturen. Die zweimonatige Lagerung bei $-20{ }^{\circ} \mathrm{C}$ war lediglich mit einem geringen Signalverlust und keiner Veränderung der Spotqualität verbunden. Somit konnte die Lagerfähigkeit des entwickelten Immunochips gezeigt werden. 


\section{Zusammenfassung und Ausblick}

Mit dem entwickelten Antigen-Immunochip können Schweineseren schnell und automatisiert auf ihren Gehalt an Antikörpern gegen das Hepatitis-E-Virus und Yersinia spp. untersucht werden. Dabei treten keine Kreuzreaktivitäten auf. Ferner ist eine Lagerung des fertigen Chips über mehrere Monate bei $-20{ }^{\circ} \mathrm{C}$ möglich.

Zukünftig soll der Immunochip um Antigene der anderen relevanten Zoonose-Erreger erweitert werden, um ein leistungsfähiges MultiplexVerfahren zu etablieren. Darüber hinaus soll die Regenerierbarkeit des Chips untersucht und optimiert werden. Erste Experimente dazu haben bereits gezeigt, dass nach 20 Regenerationszyklen immer noch etwa 20\% des Anfangssignals erhalten werden.

\section{Danksagung}

Diese Arbeit wurde von der Bayerischen Forschungsstiftung gefördert (BFS AZ-842-08).

\section{Referenzen}

[1] A. Wolter, R. Niessner, M. Seidel, Preparation and Characterization of Functional Poly(Ethylene Glycol) Surfaces for the use of Antibody Microarrays, Analytical Chemistry 79, 4529-4537 (2007); doi: 10.1021/ac070243a

[2] K. Kloth, R. Niessner, M. Seidel, Development of an Open Stand-alone Platform for Regenerable Automated Microarrays, Biosensors \& Bioelectronics 24, 2106-2112 (2009); doi: 10.1016/j.bios.2008.11.005

[3] K. Wutz, V. K. Meyer, S. Wacheck, P. Krol, M. Gareis, C. Nölting, F. Struck, E. Soutschek, O. Böcher, R. Niessner, M. Seidel, New Route for Fast Detection of Antibodies against Zoonotic Pathogens in Sera of Slaughtered Pigs by Means of Flow-through Chemiluminescence Immunochips, Analytical Chemistry 85, 52795285 (2013); doi: 10.1021/ac400781t 